\title{
TYZ expansion for the Kepler manifold 1
}

\author{
Todor Gramchev and Andrea Loi \\ Dipartimento di Matematica e Informatica - Università di Cagliari \\ Via Ospedale 72, 09124 Cagliari- Italy \\ e-mail : todor@unica.it, loi@unica.it
}

\begin{abstract}
The main goal of the paper is to address the issue of the existence of Kempf's distortion function and the Tian-Yau-Zelditch (TYZ) asymptotic expansion for the Kepler manifold - an important example of non compact manfold. Motivated by the recent results for compact manifolds we construct Kempf's distortion function and derive a precise TYZ asymptotic expansion for the Kepler manifold. We get an exact formula: finite asymptotic expansion of $n-1$ terms and exponentially small error terms uniformly with respect to the discrete quantization parameter $m \rightarrow \infty$ and $\rho \rightarrow \infty, \rho$ being the polar radius in $\mathbb{C}^{n}$. Moreover, the coefficents are calculated explicitly and they turned out to be homogeneous functions with respect to the polar radius in the Kepler manifold. We also prove and derive an asymptotic expansion of the obtstruction term with the coefficients being defined by geometrical quantities. We show that our estimates are sharp by analyzing the nonharmonic behaviour of $T_{m}$ and the error term of the approximation of the Fubini-Study metric by $m \omega$ for $m \rightarrow+\infty$. The arguments of the proofs combine geometrical methods, quantization tools and functional analytic techniques for investigating asymptotic expansions in the framework of analytic-Gevrey spaces.
\end{abstract}

Keywords: Kähler manifolds; quantization; quantum mechanics; TYZ asymptotic expansion; exponential reminder.

Subj.Class: 53C55, 58F06, 58J37

\section{Introduction and statements of the main results}

Let $g$ be a Kähler metric on a complex $n$-dimensional manifold $M$. Assume that $g$ is polarized with respect to a holomorphic line bundle $L$ over $M$, i.e. $c_{1}(L)=[\omega]$, where $\omega$ is the Kähler form associated to $g$ and $c_{1}(L)$ denote the first Chern class of $L$. Let $m \geq 1$ be a nonnegative integer and let $h_{m}$ be an Hermitian metric on $L^{m}=L^{\otimes m}$ such that its Ricci curvature $\operatorname{Ric}\left(h_{m}\right)=m \omega$. Here $\operatorname{Ric}\left(h_{m}\right)$ is the two form on $M$ whose local expression is given by

$$
\operatorname{Ric}\left(h_{m}\right)=-\frac{i}{2} \partial \bar{\partial} \log h_{m}(\sigma(x), \sigma(x)),
$$

for a trivializing holomorphic section $\sigma: U \rightarrow L^{m} \backslash\{0\}$. In the quantum mechanics terminology $L^{m}$ is called the quantum line bundle, the pair $\left(L^{m}, h_{m}\right)$ is called a geometric quantization of the Kähler manifold $(M, m \omega)$ and $h=m^{-1}$ play the role of Planck's constant (see e.g. [2]). Consider the separable complex Hilbert space $\mathcal{H}_{m}$ consisting of global holomorphic sections $s$ of $L^{m}$ such that

$$
\langle s, s\rangle_{m}=\int_{M} h_{m}(s(x), s(x)) \frac{\omega^{n}}{n !}<\infty .
$$

Let $x \in M$ and $q \in L^{m} \backslash\{0\}$ a fixed point of the fiber over $x$. If one evaluates $s \in \mathcal{H}_{m}$ at $x$, one gets a multiple $\delta_{q}(s)$ of $q$, i.e. $s(x)=\delta_{q}(s) q$. The map $\delta_{q}: \mathcal{H}_{m} \rightarrow \mathbb{C}$ is a continuous

${ }^{1}$ The first author was supported in part by GNAMPA-INDAM, Italy. The second author was supported in part by the M.I.U.R. Project "Geometric Properties of Real and Complex Manifolds". 
linear functional [9]. Hence from Riesz's theorem, there exists a unique $e_{q}^{m} \in \mathcal{H}$ such that $\delta_{q}(s)=\left\langle s, e_{q}^{m}\right\rangle_{m}, \forall s \in \mathcal{H}_{m}$, i.e.

$$
s(x)=\left\langle s, e_{q}^{m}\right\rangle_{m} q .
$$

It follows that

$$
e_{c q}^{m}=\bar{c}^{-1} e_{q}^{m}, \forall c \in \mathbb{C}^{*} .
$$

The holomorphic section $e_{q}^{m} \in \mathcal{H}_{m}$ is called the coherent state relative to the point $q$. Thus, one can define a smooth function on $M$

$$
T_{m}(x)=h_{m}(q, q)\left\|e_{q}^{m}\right\|^{2},\left\|e_{q}^{m}\right\|^{2}=\left\langle e_{q}^{m}, e_{q}^{m}\right\rangle,
$$

where $q \in L^{m} \backslash\{0\}$ is any point on the fiber of $x$. If $s_{j}, j=0, \ldots d_{m},\left(d_{m}+1=\operatorname{dim} \mathcal{H}_{m} \leq \infty\right)$ is a orthonormal basis for $\left(\mathcal{H}_{m},\langle\cdot, \cdot\rangle_{m}\right)$ then one can easily verify that

$$
T_{m}(x)=\sum_{j=0}^{d_{m}} h_{m}\left(s_{j}(x), s_{j}(x)\right) .
$$

Notice that when $M$ is compact $\mathcal{H}_{m}=H^{0}\left(L^{m}\right)$, where $H^{0}\left(L^{m}\right)$ denotes the space of global holomorphic sections of $L^{m}$. Hence in this case $d_{m}<\infty$ and (1.4) is a finite sum.

The function $T_{m}$ has appeared in the literature under different names. The earliest one was probably the $\eta$-function of J. Rawnsley [33] (later renamed to $\epsilon$ function in [9]), defined for arbitrary Kähler manifolds, followed by the distortion function of Kempf [20] and Ji [19], for the special case of Abelian varieties and of Zhang [40] for complex projective varieties. The metrics for which $T_{m}$ is constant were called critical in [40] and balanced in [13] (see also [3], 24], 26] and [27]). If $T_{m}$ are constants for all sufficiently large $m$ then the geometric quantization $\left(L^{m}, h_{m}\right)$ associated to the Kähler manifold $(M, g)$ is called regular. Regular quantization play a prominent role in the theory of quantization by deformation of Kähler manifolds developed in [9] (see also [23]).

Fix $m \geq 1$. Under the hypothesis that for each point $x \in M$ there exists $s \in \mathcal{H}_{m}$ non-vanishing at $x$, one can give a geometric interpretation of $T_{m}$ as follows. Consider the holomorphic map of $M$ into the complex projective space $\mathbb{C} P^{d_{m}}$ :

$$
\varphi_{m}: M \rightarrow \mathbb{C} P^{d_{m}}: x \mapsto\left[s_{0}(x): \cdots: s_{d_{m}}(x)\right]
$$

One can prove that

$$
\varphi_{m}^{*}\left(\omega_{F S}\right)=m \omega+\frac{i}{2} \partial \bar{\partial} \log T_{m}
$$

where $\omega_{F S}$ is the Fubini-Study form on $\mathbb{C} P^{d_{m}}$, namely the form which in homogeneous coordinates $\left[Z_{0}, \ldots, Z_{d_{m}}\right]$ reads as $\omega_{F S}=\frac{i}{2} \partial \bar{\partial} \log \sum_{j=0}^{d_{m}}\left|Z_{j}\right|^{2}$.

Clearly (1.6) leads to

$$
\frac{\varphi_{m}^{*}\left(\omega_{F S}\right)}{m}-\omega=\frac{i}{2 m} \partial \bar{\partial} \log T_{m}
$$

therefore the term

$$
\mathcal{E}_{m}(x):=\frac{i}{2 m} \partial \bar{\partial} \log T_{m},
$$

turns out to play a role of the "error" of the approximation of $\omega$ (resp. $g$ ) by $\frac{\varphi_{m}^{*}\left(\omega_{F S}\right)}{m}$ (resp. $\left.\frac{\varphi_{m}^{*}\left(g_{F S}\right)}{m}\right)$. 
Observe that by (1.6), if there exists $m$ such that $m g$ is a balanced metric, or more generally if $T_{m}$ is harmonic, then $\mathcal{E}_{m}(x)$ is identically zero and hence $m g$ is projectively induced via the coherent states map $\varphi_{m}$ (see [2] for more details on the link between projectively induced Kähler metrics and balanced metrics). Recall that a Kähler metric $g$ on a complex manifold $M$ is projectively induced if there exists a Kähler (i.e. a holomorphic and isometric) immersion $\psi: M \rightarrow \mathbb{C} P^{N}, N \leq \infty$ such that $\psi^{*}\left(g_{F S}\right)=g$. Projectively induced Kähler metrics enjoyes very nice properties and they were deeply studied in [8] (see also the begining of Section 4 below). Not all Kähler metrics are balanced or projectively induced. Nevertheless, when $M$ is compact, Tian [38] and Ruan [34] solved a conjecture posed by Yau by proving that the sequence of metrics $\frac{\varphi_{m}^{*}\left(\omega_{F S}\right)}{m} C^{\infty}$-converges to $\omega$. In other words, any polarized metric on compact complex manifold is, the $C^{\infty}$-limit of (normalized) projectively induced Kähler metrics. Zelditch [39] generalized Tian-Ruan theorem by proving a complete asymptotic expansion in the $C^{\infty}$ category, namely

$$
T_{m}(x) \sim \sum_{j=0}^{\infty} a_{j}(x) m^{n-j}
$$

where $a_{j}, j=0,1, \ldots$, are smooth coefficients with $a_{0}(x)=1$, and for any nonnegative integers $r, k$ the following estimates hold:

$$
\left\|T_{m}(x)-\sum_{j=0}^{k} a_{j}(x) m^{n-j}\right\|_{C^{r}} \leq C_{k, r} m^{n-k-1},
$$

where $C_{k, r}$ are constant depending on $k, r$ and on the Kähler form $\omega$ and $\|\cdot\|_{C^{r}}$ denotes the $C^{r}$ norm in local coordinates..

Later on, Lu [28, by means of Tian's peak section method, proved that each of the coefficients $a_{j}(x)$ in (1.9) is a polynomial of the curvature and its covariant derivatives at $x$ of the metric $g$. Such a polynomials can be found by finitely many steps of algebraic operations. Furthermore $a_{1}(x)=\frac{1}{2} \rho$, where $\rho$ is the scalar curvature of the polarized metric $g$ (see also [21] and [22] for the computations of the coefficients $a_{j}$ 's through Calabi's diastasis function).

The expansion (1.9) is called the TYZ (Tian-Yau-Zelditch) expansion.

The aim of the present paper is to adress the problem of TYZ expansions for noncompact manifolds. Our motivations is twofolded. First, its is purely geometrical question of its own interest. Secondly, we are inspired by the previous works of M. Engliš [16], [17], [18], where analytical tools from the the theory of asymptotic expansions have been applied in order to extend Berezin's quantization method cf. [5], 6] to non homogeneous complex domains on $\mathbb{C}^{n}$ (see also [29], [30], 31]).

We choose as a noncompact manifold the Kepler manifold $(X, \omega)$, namely the cotangent bundle of the $n$-dimensional sphere minus its zero section endowed with the standard symplectic form $\omega$ (see [37] and [32]). This manifold has been considered by different authors and we bilieve our results can be of some interest both from the mathematical and physical point of view.

We summarize the main novelties of our work: First, we compute explicitly the Kempf distortion function $T_{m}(x)$ for the Kepler manifold $(X, \omega)$. Secondly, based on this computation we find an analogue of Zelditch and Lu's theorems above for $(X, \omega)$. More precisely, building upon the explicit representation of $T_{m}$ as an action of "singular derivatives" and 
using precise analytical methods pertinent to the study of nonlinear compositions in functional spaces, we show that the TYZ expansion for the Kepler manifold has two remarkable features in comparison with the known results for compact manifolds:

- first, the TYZ expansion is finite. More precisely, it consists of $n-1$ terms

$$
T_{m}(x)=m^{n}+\frac{(n-2)(n-1)}{2|x|} m^{n-1}+\sum_{k=2}^{n-2} \frac{2 a_{k}}{|x|^{k}} m^{n-k}+R_{m}(|x|),
$$

where $a_{k}, k \geq 2$ can be computed explicitely by recursive formulas.

- secondly, the reminder term has an exponential small decay $O\left(e^{-c m}\right)$ as $m \rightarrow \infty$ uniformly with respect to $|x| \geq \delta>0$. We point out that our exact formula modulo exponentially small error for Kempf's distortion function might be viewed as an analogue to a geometric interpretation of exact asymptotic formulas appearing for the moment map and equivariant cohomology cf. M. F. Attiyah and R. Bott [4] (see also [14], [15]).

We also derive uniform analytic-Gevrey estimates for $T_{m}$ keeping the exponential decay for $m \rightarrow \infty,|x| \rightarrow \infty$ which resemble the estimates in the framework of Gelfand-Shilov spaces $S_{1}^{1}$ appearing in the regularity theory for pseudodifferential operators cf. [10], [11]. Observe that as for the compact case our expansion shows that $g$ (the metric $g$ associated to the Kepler manifold $(M, \omega))$ is the $C^{\infty}$-limit of (suitable normalized) projectively induced Kähler metrics, namely $\lim _{m \rightarrow \infty} \frac{1}{m} \varphi_{m}^{*}\left(g_{F S}\right)=g$ where $\varphi_{m}: X \rightarrow \mathbb{C} P^{\infty}$ is the coherent states map. A geometric construction is proposed showing that our estimates are sharp. Indeed we show that $g$ is not projectively induced, i.e. it cannot exist any Kähler immersion of $(X, \omega)$ into a finite or infinite dimensional complex projective space. The arguments use Calabi's tools which provide necessary and sufficient conditions for a Kähler metric to be projecticely induced.

Finally, we investigate the asymptotic behaviour of the obstruction term

$$
\mathcal{E}_{m}(z)=\sum_{j, \ell=1}^{n+1} \mathcal{E}_{m}^{j, \ell}(z) d z_{j} \wedge d \bar{z}_{\ell}
$$

in (1.8) and prove that the coefficients decay polynomially of the type $m^{-2}$. More precisely, for some $C>0$, they behave like

$$
\frac{C}{m^{2}|z|^{3}}(1+o(1)) \quad m \rightarrow \infty
$$

uniformly for $|z|$ away from the origin in $\mathbb{C}^{n}$. In fact, we show an abstract theorem for the asymptotic behaviour of obstruction terms similar to (1.8) on conic manifolds of Kepler type. The proof is based on a suitable choice of global singular coordinates parametrizing the Kepler manifold and the use of implicit function theorem arguments. Consequently, by (1.6), the metric $g$ associated to $\omega$ can be approximated by suitable normalized projectively induced Kähler metrics with an error of the type $m^{-2}, m \rightarrow \infty$.

The paper is organized as follows. We propose an explicit construction of the Kempf distortion function $T_{m}$ for the Kepler manifold $(X, \omega)$ in Section 2. In Section 3 we derive an exact TYZ asymptotic expansion and derive the exponentially small decay for the remainder 
when $m \rightarrow \infty$. In Section 4 we prove (see Theorem 4.4) that our estimate is sharp. Finally, Section 5 contains the construction of the global singular parametrization of the Kepler manifold and the study of the asymptotic behaviour of the logarithmic obstruction term (1.8).

\section{Kempf's distortion function for the Kepler manifold}

The (regularized) Kepler manifold [37] is (may be identifed with) the $2 n$-dimensional symplectic manifold $(X, \omega)$, where $X=T^{*} S^{n} \backslash 0$ the cotangent bundle to the $n$-dimensional sphere minus its zero section endowed with the standard symplectic form $\omega$. This may further be identified with

$$
X=\left\{(e, x) \in \mathbb{R}^{n+1} \times \mathbb{R}^{n+1} \mid e \cdot e=1, x \cdot e=0, x \neq 0\right\}
$$

where the dot denotes the standard scalar product on $\mathbb{R}^{n+1}$. In [37] J. Souriau showed that the Kepler manifold admits a natural complex structure. Indeed he proved that by introducing

$$
z=|x| e+i x \in \mathbb{C}^{n+1}=|x|(e+i s), \quad s=\frac{x}{|x|} \in S^{n},
$$

then $X$ is diffeomorphic to the isotropic cone

$$
C=\left\{z \in \mathbb{C}^{n+1} \mid z \cdot z=z_{1}^{2}+\cdots+z_{n+1}^{2}=0, z \neq 0\right\} \subset \mathbb{C}^{n+1}
$$

and hence $X$ inherits the complex structure of $C$ via this diffeomorphism. Seven years later J. Rawnsley [33] observed that the symplectic form $\omega$ is indeed a Kähler form with respect to this complex structure and it can be written (up to a factor) as

$$
\omega=\frac{i}{2} \partial \bar{\partial}|x|
$$

Moreover, since $\omega$ is exact, it is trivially integral and hence there exists a holomorphic line bundle $L$ over $X$ such that $c_{1}(L)=[\omega]$.

For $n \geq 3, X$ is simply-connected so $L^{m}$ is holomorphically trivial $\left(L^{m}=X \times \mathbb{C}\right)$ and we can identify $H^{0}\left(L^{m}\right)$ with the set of holomorphic functions of $X$. Furthermore, we can define an Hermitian metric $h_{m}$ on $L^{m}=X \times \mathbb{C}$ by

$$
h_{m}(\sigma(z), \sigma(z))=e^{-m|x|}
$$

where $\sigma: X \rightarrow X \times \mathbb{C}$, is the global holomorphic section such that $\sigma(z)=(z, 1)$. It follows by (1.1) above that the pair $\left(L^{m}, h_{m}\right)$ is indeed a geometric quantization of the Kepler manifold $(X, \omega)$. Then the Hilbert space $\mathcal{H}_{m}$ consists of the set of homorphic functions $f$ of $X$ such that

$$
\|f\|_{m}^{2}:=\int_{X}|f(z)|^{2} e^{-m|x|} d \mu(z)<\infty
$$

where

$$
d \mu(z)=\frac{\omega^{n}(z)}{n !}=\left(\frac{i}{2} \partial \bar{\partial}|x|\right)^{n} .
$$

Notice that in this case

$$
T_{m}(z)=e^{-m|x|} K^{(m)}(z, z),
$$


where $K^{(m)}(z, z)$ is the reproducing Kernel for the Hilbert space $\mathcal{H}_{m}$. At p. 412 in 33 Rawnsley explicitly computed $K(z, z)=K^{(1)}(z, z)$ (the reproducing kernel for $\mathcal{H}=\mathcal{H}_{1}$ ) and hence the corresponding Kempf's distortion function, which in our notations reads as:

$$
T_{1}(z)=e^{-|x|} \mathrm{K}(z, z)=2^{n-1} e^{-|x|} \sum_{j=0}^{\infty} \frac{(j+n-2) !}{(2 j+n-2) !} \frac{|x|^{2 j}}{j !}, 2|x|^{2}=z \cdot \bar{z} .
$$

Now, we compute the Kempf distortion functions $T_{m}(z)$ for all non-negative integers $m$ as follows. Making the change of variable $m z=w$, we get

$$
\|f\|_{m}^{2}=\int_{X}|f(w / m)|^{2} e^{-|\operatorname{Im} w|} m^{-n} d \mu(w)
$$

since $d \mu(w / m)=m^{-n} d \mu(w)$. Consequently, the operator

$$
T: T f(w):=m^{-\frac{n}{2}} f(w / m)
$$

is a unitary isomorphism from $\mathcal{H}_{m}$ onto $\mathcal{H} 2$. Denoting by $\mathrm{K}^{(m)}(w, z) \equiv \mathrm{K}_{z}^{m}(w)$ the reproducing kernel of $\mathcal{H}_{m}$ (and writing simply $\mathrm{K}(w, z) \equiv K_{z}(w)$ if $m=1$ ), we therefore have, on the one hand,

$$
f(z)=\left\langle f, \mathrm{~K}_{z}^{(m)}\right\rangle_{m}=\left\langle T f, T \mathrm{~K}_{z}^{(m)}\right\rangle
$$

for any $f \in \mathcal{H}_{m}$, while, on the other hand,

$$
f(z)=m^{\frac{n}{2}} T f(m z)=\left\langle T f, m^{\frac{n}{2}} \mathrm{~K}_{m z}\right\rangle .
$$

Thus $T \mathrm{~K}_{z}^{(m)}=m^{\frac{n}{2}} \mathrm{~K}_{m z}$, and

$$
\mathrm{K}_{z}^{(m)}(w)=m^{\frac{n}{2}} T^{-1} \mathrm{~K}_{m z}(w)=m^{n} \mathrm{~K}_{m z}(m w) .
$$

That is,

$$
\mathrm{K}^{(m)}(w, z)=m^{n} \mathrm{~K}(m w, m z) .
$$

Substituting this into Rawsley's formula (2.14), we thus get

$$
T_{m}(z)=e^{-m|x|} \mathrm{K}^{(m)}(z, z)=2^{n-1} m^{n} e^{-m|x|} \sum_{j=0}^{\infty} \frac{(j+n-2) !}{(2 j+n-2) !} \frac{(m|x|)^{2 j}}{j !}
$$

Remark 2.1 From (2.15) one sees that $T_{m}(x)=m^{n} T_{1}(m x)$. In the compact case the relationship between $T_{m}$ and $T_{1}$ is unknown. Here the fact that the Hilbert spaces involved are infinite dimensional is a crucial step to get the previous equality.

Notice that from representation (2.15) is not clear the growth of $T_{m}(z)$ as $m \rightarrow \infty$. The following proposition gives us important analytic information about $T_{m}$ as $m \rightarrow \infty$.

\footnotetext{
${ }^{2}$ The second author is in debt with Miroslav Engliš who pointed him out the idea of using this isomorphism to compute $T_{m}$ from $T_{1}$.
} 
Proposition 2.1 Kempf's distortion function for the Kepler manifold can be written in the following two forms:

$$
T_{m}(z)=2^{-1} m^{n} e^{-m|x|} \sum_{j=0}^{\infty}\left(1+\tau_{j}\right) \frac{(m|x|)^{2 j}}{(2 j) !}
$$

where

$$
\tau_{j}=1-\frac{(j+1) \ldots(j+n-2)}{j+1 / 2) \ldots(j+(n-2) / 2} \longrightarrow 0 \quad \text { for } j \rightarrow \infty
$$

and

$$
T_{m}(z)=2 m^{n} e^{-\xi_{m}}\left(\frac{1}{\xi_{m}} \frac{\partial}{\partial \xi_{m}}\right)^{n-2}\left[\xi_{m}^{n-2}\left(e^{\xi_{m}}+(-1)^{n-2} e^{-\xi_{m}}+Q\left(\xi_{m}\right)\right)\right],
$$

where $\xi_{m}=m|x|, Q\left(\xi_{m}\right)$ is a polynomial of degree $\leq n-4$ in the variable $\xi_{m}$.

Proof. From (2.15) one gets

$$
\begin{aligned}
T_{m}(z) & =e^{-m|x|} K^{(m)}(z, z)=2^{n-1} m^{n} e^{-m|x|} \sum_{j=0}^{\infty} \frac{(j+n-2) !}{(2 j+n-2) !} \frac{(m|x|)^{2 j}}{j !} \\
& =2^{n-1} m^{n} e^{-m|x|} \sum_{j=0}^{\infty} \frac{(j+n-2) !(2 j) !}{j !(m-n+x \mid)^{2 j}} \\
& =2^{-1} m^{n} e^{-m|x|} \sum_{j=0}^{\infty} \frac{(j+1) \ldots(j+n-2)}{(2 j) !} \frac{(m|x|)^{2 j}}{(2 j) !} \\
& =2^{-1} m^{n} e^{-m|x|} \sum_{j=0}^{\infty}\left(1+\tau_{j}\right) \frac{(m|x|)^{2 j}}{(2 j) !}
\end{aligned}
$$

In order to prove (2.17) set

$$
y_{m}^{2}=m|x|=\xi_{m}, y_{m}, \xi_{m} \in \mathbb{R} \backslash\{0\} .
$$

Then, since $\frac{\partial}{\partial y_{m}}=\frac{1}{2 \xi_{m}} \frac{\partial}{\partial \xi_{m}}$ one gets

$$
\begin{aligned}
T_{m}(z) & =2^{n-1} m^{n} e^{-\xi_{m}} \sum_{j=0}^{\infty} \frac{(j+n-2) !}{(2 j+n-2) !} \frac{y_{m}^{j}}{j !} \\
& =2^{n-1} m^{n} e^{-\xi_{m}}\left(\frac{\partial}{\partial y_{m}}\right)^{n-2} \sum_{j=0}^{\infty} \frac{y_{m}^{j+n-2}}{(2 j+n-2) !} \\
& =2 m^{n} e^{-\xi_{m}}\left(\frac{1}{\xi_{m}} \frac{\partial}{\partial \xi_{m}}\right)^{n-2}\left[\xi_{m}^{n-2} \sum_{j=0}^{\infty} \frac{\xi_{m}^{2 j+n-2}}{(2 j+n-2) !}\right]
\end{aligned}
$$

If $n$ is even then

$$
T_{m}(z)=2 m^{n} e^{-\xi_{m}}\left(\frac{1}{\xi_{m}} \frac{\partial}{\partial \xi_{m}}\right)^{n-2}\left[\xi_{m}^{n-2}\left(\cosh \xi_{m}-P\left(\xi_{m}\right)\right)\right],
$$

where

$$
P\left(\xi_{m}\right)=\sum_{j=0}^{\frac{n-4}{2}} \frac{\xi_{m}^{2 j}}{(2 j) !} .
$$

If $n$ is odd then

$$
T_{m}(z)=2 m^{n} e^{-\xi_{m}}\left(\frac{1}{\xi_{m}} \frac{\partial}{\partial \xi_{m}}\right)^{n-2}\left[\xi_{m}^{n-2}\left(\sinh \xi_{m}-R\left(\xi_{m}\right)\right)\right],
$$


where

$$
R\left(\xi_{m}\right)=\sum_{j=0}^{\frac{n-5}{2}} \frac{\xi_{m}^{2 j+1}}{(2 j+1) !}
$$

and hence (2.17) easily follows.

Remark 2.2 By (2.16) one might view $T_{m}(z)$ as a "small perturbation" (or close to) for $m \rightarrow \infty$ of

$$
T_{m}^{0}(z)=2^{-1} m^{n} e^{-m|x|} \sum_{j=0}^{\infty} \frac{(m|x|)^{2 j}}{(2 j) !}=m^{n} e^{-m|x|} \cosh (m|x|)=m^{n} \frac{1-e^{-2 m|x|}}{2} .
$$

\section{TYZ expansion for the Kepler manifold}

The key ingredient to find the TYZ expansion of $T_{m}$ for the Kempf distortion function of the Kepler manifold is (2.17). Clearly we have

$$
T_{m}(z)=2 m^{n} F(m|x|),
$$

where

$$
F(y)=e^{-y}\left(\frac{1}{y} \frac{d}{d y}\right)^{n-2}\left(y^{n-2}\left(e^{y}+(-1)^{n-2} e^{-y}+Q(y)\right)\right), \quad y \in \mathbb{R} .
$$

The explicit representation (3.19)-(3.20) of $T_{m}(z)$ for the Kepler manifold has a remarkable feature, namely, it is defined by a generating function $F(y)$ depending on one variable. Note that in fact $T_{m}(z)$ is independent of the base variables $e \in S^{n}$.

The first main result of the present paper is the following one.

Theorem 3.1 Let F satisfy (3.20). Then the following representation holds:

$$
F(y)=\sum_{j=0}^{n-2} \frac{b_{j}}{y^{j}}+\Phi(y)+\Psi(y)
$$

where

$$
\begin{aligned}
& \Phi(y)=e^{-2 y} \sum_{j=0}^{n-2} \frac{p_{j}}{y^{j}} \\
& \Psi(y)=e^{-y} \sum_{j=0}^{n-3} \frac{r_{j}}{y^{j}}
\end{aligned}
$$

and the constants $a_{j}, p_{j}, r_{j}$ are calculated explicitly. The functions $\Phi(y), \Psi(y)$ and therefore, $F(y)$ as well, are extended to holomorphic functions in semiplane Rey $>0$. In particular, by (3.19) and (3.21) we get

$$
T_{m}(z)=\sum_{j=0}^{n-2} a_{j}(x) m^{n-j}+2 m^{n} \Phi(m|x|)+2 m^{n} \Psi(m|x|), m \in \mathbb{N},
$$


where

$$
a_{j}(x)=\frac{2 b_{j}}{|x|^{j}}, \quad j=0,1, \ldots, n-2 .
$$

and

$$
\begin{aligned}
& a_{0}(x)=1 \\
& a_{1}(x)=\frac{(n-2)(n-1)}{2|x|}
\end{aligned}
$$

Moreover, there exists an absolute constant $C_{0}>0$ such that for every $\left.\left.\delta \in\right] 0,1\right]$

$$
\sup _{|x| \geq \delta}\left|D_{x}^{\alpha} \Theta_{m}(x)\right|, \leq C_{0}^{\alpha+1} \frac{\alpha !}{\delta^{\alpha}} e^{-m \delta / 2}
$$

for all $m \in \mathbb{N}$, where $\Theta=\Phi, \Psi$. Therefore, we have the following estimates

$$
\left|D_{x}^{\alpha}\left(T_{m}-\sum_{j=0}^{n-2} a_{j}(x) m^{n-j}\right)\right| \leq C_{0}^{\alpha+1} \frac{\alpha !}{\delta^{\alpha}} e^{-m \delta / 2}
$$

for all $|x| \geq \delta, \alpha \in \mathbb{Z}_{+}^{n}$.

Proof. We recall the well known Faà di Bruno type formula for the derivative of $g \circ \varphi$, namely, for a given $\alpha \in \mathbb{N}$ we have

$$
\begin{aligned}
D_{t}^{\alpha}(g(\varphi(t))) & =\left.D_{y}^{\alpha}(g(\varphi(y)))\right|_{y=t} \\
& =\left.\sum_{j=1}^{\alpha} \frac{g^{(j)}(\varphi(t))}{j !} D_{x}^{\alpha}\left((\varphi(x)-\varphi(t))^{j}\right)\right|_{x=t} \\
& =\sum_{j=1}^{\alpha} \frac{g^{(j)}(\varphi(t))}{j !} \sum_{\substack{\alpha_{1}+\ldots+\alpha_{j}=\alpha \\
\alpha_{1} \geq 1, \ldots, \alpha_{j} \geq 1}} \frac{\alpha !}{\alpha_{1} ! \ldots \alpha_{j} !} \varphi^{\left(\alpha_{1}\right)}(t) \ldots \varphi^{\left(\alpha_{j}\right)}(t),
\end{aligned}
$$

where $\varphi^{(k)}(t)$ stands for $D_{t}^{k} \varphi(t)$.

Next, we straighten $y^{-1} D_{y}$ into $D_{t}$ via the change of the variable $y=y(t)=\sqrt{2 t}$, $t=t(y)=y^{2} / 2$. Therefore, setting

$$
G(t)=F(\sqrt{2 t}), t>0, \quad F(y)=G\left(\frac{y^{2}}{2}\right), y>0
$$

we get by (3.20)

$$
F(y)=G(t)=e^{-\sqrt{2 t}}\left(\frac{d}{d t}\right)^{n-2}\left((2 t)^{(n-2) / 2} \frac{e^{\sqrt{2 t}}+(-1)^{n-2} e^{-\sqrt{2 t}}}{2}+Q(\sqrt{2 t})\right) .
$$

The next assertion is instrumental in the proof. 
Lemma 3.1 Let $N \in \mathbb{N}, c \in \mathbb{R}$, and $r>0$. Then

$$
\begin{aligned}
\psi_{N}^{c, r}(y) & :=e^{-y}\left(\frac{1}{y} \frac{d}{d y}\right)^{N}\left(y^{r} e^{c y}\right) \\
& =\psi_{N}^{c, r}(\sqrt{2 t})=: \varphi_{N}^{c, r}(t)=e^{-\sqrt{2 t}}\left(\frac{d}{d t}\right)^{N}\left((2 t)^{r / 2} e^{c \sqrt{2 t}}\right) .
\end{aligned}
$$

has the following representation

$$
\varphi_{N}^{c, r}(t)=e^{-(1-c) \sqrt{2 t}}(2 t)^{(r-N) / 2} \sum_{s=0}^{N} \frac{\varkappa_{s}}{(2 t)^{s / 2}},
$$

i.e.

$$
\psi_{N}^{c, r}(t)=e^{-(1-c) z} z^{(r-N) / 2} \sum_{s=0}^{N} \frac{\varkappa_{s}}{z^{s}},
$$

where

$$
\begin{aligned}
\varkappa_{s} & =\frac{1}{(N-s) !} \sum_{\ell=N-s}^{N}\left(\begin{array}{c}
N \\
\ell
\end{array}\right)\left(\prod_{q=0}^{N-\ell-1}\left(\frac{r}{2}-q\right)\right) 2^{N-r / 2}(-1)^{\ell+s-N} \\
& \times \sum_{\substack{\ell_{1}+\ldots+\ell_{N-s}=\ell \\
\ell_{1} \geq 1, \ldots, \ell_{N-s} \geq 1}} \frac{\ell !}{\ell_{1} ! \ldots \ell_{N-s} !} \prod_{q_{1}}^{\ell_{1}-1}\left(\frac{1}{2}-q_{1}\right) \ldots \prod_{q_{N-s}}^{\ell_{N-s}-1}\left(\frac{1}{2}-q_{N-s}\right)
\end{aligned}
$$

for $s=0, \ldots, N-1$ and

$$
\varkappa_{N}=2^{N-r / 2} \prod_{q=0}^{N-1}\left(\frac{r}{2}-q\right) .
$$

Proof. By Faà di Bruno type formula (3.30) we derive

$$
\begin{aligned}
\Theta_{N}^{r, c}(t) & =\left(\frac{d}{d t}\right)^{N}\left(t^{r / 2} e^{c \sqrt{2 t}}\right) \\
& =\sum_{\ell=0} N\left(\begin{array}{c}
N \\
\ell
\end{array}\right) D_{t}^{N-\ell}\left(t^{r / 2}\right) D_{t}^{\ell}\left(e^{c \sqrt{2 t}}\right) \\
& =D_{t}^{N}\left(t^{r / 2}\right) e^{c \sqrt{2 t}}+\sum_{\ell=1} N\left(\begin{array}{c}
N \\
\ell
\end{array}\right)\left(\prod_{q=0}^{N-\ell-1}\left(\frac{r}{2}-q\right)\right) t^{r / 2-N+\ell} e^{c \sqrt{2 t}} \sum_{j=1}^{\ell} \frac{(c 2)^{j / 2}}{j !} \\
& \times \sum_{\substack{\ell_{1}+\cdots+\ell_{j}=\ell \\
\ell_{1} \geq 1, \ldots, \ell_{j} \geq 1}} \frac{\ell !}{\ell_{1} ! \ldots \ell_{j} !} D_{t}^{\ell_{1}}\left(t^{1 / 2}\right) \ldots D_{t}^{\ell_{j}}\left(t^{1 / 2}\right)
\end{aligned}
$$

with the convention $\prod_{q=0}^{-1} \ldots=1$. Since

$$
\begin{aligned}
D_{t}^{\mu}\left(t^{1 / 2}\right) & =\frac{1}{2}\left(\frac{1}{2}-1\right) \ldots\left(\frac{1}{2}-\mu+1\right) t^{1 / 2-\mu} \\
& =(-1)^{\mu-1} \frac{(2 \mu-3) ! !}{2^{\mu}} t^{1 / 2-\mu}
\end{aligned}
$$


for all positive integers $\mu$, with $(-1) ! !:=1,(2 \mu-3) ! !:=1 \ldots(2 \mu-3)$ if $\mu \geq 2$, combining (3.38) and (3.39), we obtain

$$
\sum_{\substack{\ell_{1}+\cdots+\ell_{j}=\ell \\ \ell_{1} \geq 1, \ldots, \ell_{j} \geq 1}} \frac{\ell !}{\ell_{1} ! \ldots \ell_{j} !} D_{t}^{\ell_{1}}\left(t^{1 / 2}\right) \ldots D_{t}^{\ell_{j}}\left(t^{1 / 2}\right)=(-1)^{\ell-j} \Gamma^{\ell, j} \frac{(2 t)^{j / 2-\ell}}{2^{j / 2}}
$$

with

$$
\Gamma^{\ell, j}:=\sum_{\substack{\ell_{1}+\cdots+\ell_{j}=\ell \\ \ell_{1} \geq 1, \ldots, \ell_{j} \geq 1}} \frac{\ell !}{\ell_{1} ! \ldots \ell_{j} !}\left(2 \ell_{1}-3\right) ! ! \ldots\left(2 \ell_{j}-3\right) ! !
$$

We note that

$$
\begin{aligned}
\Gamma^{\ell, \ell} & =\ell ! \\
\Gamma^{\ell, \ell-1} & =-\frac{\ell-1}{2} \ell !
\end{aligned}
$$

Therefore, by (3.38) - (3.40),

$$
\begin{aligned}
& \Theta_{N}^{r, c}(t)=2^{N-r / 2}\left(\prod_{q=0}^{N-1}\left(\frac{r}{2}-q\right)\right)(2 t)^{r / 2-N} e^{c \sqrt{2 t}} \\
& +\sum_{\ell=1}^{N}\left(\begin{array}{c}
N \\
\ell
\end{array}\right)\left(\prod_{q=0}^{N-\ell-1}\left(\frac{r}{2}-q\right)\right) 2^{N-\ell-r / 2}(2 t)^{r / 2-N+\ell} \\
& \times \sum_{j=1}^{\ell} \frac{c^{j / 2}}{j !}(-1)^{\ell-j} \Gamma^{\ell, j}(2 t)^{j / 2-\ell} \\
& =(2 t)^{r / 2-N / 2} e^{c \sqrt{2 t}} \frac{2^{N-r / 2} \prod_{q=0}^{N-1}\left(\frac{r}{2}-q\right)}{(2 t)^{N / 2}} \\
& +(2 t)^{r / 2-N / 2} e^{c \sqrt{2 t}} \sum_{j=1}^{N} \frac{1}{j !(2 t)^{(N-j) / 2}} \sum_{\ell=j}^{N}\left(\begin{array}{c}
N \\
\ell
\end{array}\right)\left(\prod_{q=0}^{N-\ell-1}\left(\frac{r}{2}-q\right)\right) 2^{N-\ell-r / 2}(-1)^{\ell-j} \Gamma^{\ell, j} \\
& =(2 t)^{r / 2-N / 2} e^{c \sqrt{2 t}} \frac{2^{N-r / 2} \prod_{q=0}^{N-1}\left(\frac{r}{2}-q\right)}{(2 t)^{N / 2}} \\
& +(2 t)^{r / 2-N / 2} e^{c \sqrt{2 t}} \sum_{s=0}^{N-1} \frac{1}{(N-s) !(2 t)^{s / 2}} \sum_{\ell=N-s}^{N}\left(\begin{array}{c}
N \\
\ell
\end{array}\right) \\
& \times\left(\prod_{q=0}^{N-\ell-1}\left(\frac{r}{2}-q\right)\right) 2^{N-\ell-r / 2}(-1)^{\ell+s-N} \Gamma^{\ell, N-s} \\
& =(2 t)^{r / 2-N / 2} e^{c \sqrt{2 t}} \sum_{s=0}^{N} \frac{\varkappa_{s}}{(2 t)^{s / 2}}
\end{aligned}
$$

where $\varkappa_{s}$ is defined by

$$
\varkappa_{s}:=\frac{1}{(N-s) !} \sum_{\ell=N-s}^{N}\left(\begin{array}{c}
N \\
\ell
\end{array}\right)\left(\prod_{q=0}^{N-\ell-1}\left(\frac{r}{2}-q\right)\right) 2^{N-\ell-r / 2}(-1)^{\ell+s-N} \Gamma^{\ell, N-s}
$$


In view of the definition of $\Gamma^{\ell, j}$ with the convention $\Gamma^{\ell, 0}=1$, it is equivalent to (3.36), (3.37). This ends the proof of the lemma.

We conclude the proof of the theorem by applying the previous lemma for $z=m|x|$ and obtain the value of $a_{s}=1 / 2 \varkappa_{s}^{N, r ; c}$ by setting $c=1, r=N=(n-2) ; p_{s}=(-1)^{n-2} / 2 \varkappa_{s}^{N, r ; c}$ by setting $c=-1, r=N=n-2$; and

$$
r_{s}=\sum_{j=0}^{n-3} q_{j} \varkappa^{n-2, j ; 0}
$$

provided $Q(z)=\sum_{j=0}^{n-3} q_{j} z^{j}$

Remark 3.2 In view of (3.24), we have

$$
T_{m}(z)=m^{n}+\frac{(n-2)(n-1)}{2|x|} m^{n-1}+\sum_{k=2}^{n-2} \frac{2 a_{k}}{|x|^{k}} m^{n-k}+R_{m}(|x|),
$$

with $R_{m}(x)$ being exponentially small $e^{-c m}$ away from the origin $x=0$.

Remark 3.3 The novelty of the theorem above is twofolded. First, our TYZ type expansion is finite, i.e., $a_{j}=0$ for $j \geq n-1$ (compare (1.10)). Secondly, the reminder is exponentially small. Moreover, the coefficients $a_{j}$ can be computed explicitely. In a forthcoming paper we study tha link between these coefficients $a_{j}$ and the curvature of the metric $g$ as in Lu's Theorem [28].

Remark 3.2 One can also investigate the asymptotic expansion near the singular (conic) point. Using a local coordinates in which it coincides with the origin, we can derive explicit asymptotic expansion for Kempf's distortion function $T_{m}(x)$ near $x=0$. Moreover one can show that

$$
\left.\left\|T_{m}(x)-\sum_{j=0}^{n-2} a_{j}(x) m^{n-j}\right\|_{L^{p}(B(\delta))}=O\left(\delta^{n-(n-2) p}\right)\right) m^{2}, \quad \delta \searrow 0, m \geq 1
$$

provided $1 \leq p<n /(n-2)$. So we encounter the critical $L^{p}$ index which appears in different mathematical problems.

\section{Proof that our estimate is sharp}

As a consequence of Theorem 3.1 and 1.7 the Kähler form $g$ on the Kepler manifold $X$ is the $C^{\infty}$-limit of suitable normalized projectively induced Kähler metrics, namely

$$
\lim _{m \rightarrow \infty} \frac{1}{m} \varphi_{m}^{*}\left(g_{F S}\right)=g
$$

where $\varphi_{m}: X \rightarrow \mathbb{C} P^{\infty}$ is the coherent states map. In this Section we show that $g$ is not projectively induced (via any map) and then that our extimate in Theorem 3.1 is sharp. 
We need to recall briefly some results about Calabi's diastasis function referring the reader to [8] and [25] for details and further results.

Let $M$ be a complex manifold endowed with a real analytic Kähler metric $g$. Then, in a neighborhood of every point $p \in M$, one can introduce a very special Kähler potential $D_{p}^{g}$ for the Kähler form $\omega$ associated to $g$, which Calabi [8] christened diastasis. Recall that a Kähler potential is an analytic function $\Phi$ defined in a neighborhood of a point $p$ such that $\omega=\frac{i}{2} \bar{\partial} \partial \Phi$. A Kähler potential is not unique: it is defined up to an addition with the real part of a holomorphic function. By duplicating the variables $z$ and $\bar{z}$ a potential $\Phi$ can be complex analytically continued to a function $\tilde{\Phi}$ defined in a neighborhood $U$ of the diagonal containing $(p, \bar{p}) \in M \times \bar{M}$ (here $\bar{M}$ denotes the manifold conjugated to $M$ ). The diastasis function is the Kähler potential $D_{p}^{g}$ around $p$ defined by

$$
D_{p}^{g}(q)=\tilde{\Phi}(q, \bar{q})+\tilde{\Phi}(p, \bar{p})-\tilde{\Phi}(p, \bar{q})-\tilde{\Phi}(q, \bar{p}) .
$$

Observe that the diastasis does not depend on the potential chosen, $D_{p}^{g}(q)$ is symmetric in $p$ and $q$ and $D_{p}^{g}(p)=0$.

The diastasis function is the key tool for studying the Kähler immersions of a Kähler manifold into another Kähler manifold as expressed by the following lemma.

Lemma 4.1 (Calabi [8]) Let $(M, g)$ be a Kähler manifold which admits a Kähler immersion $\varphi:(M, g) \rightarrow(S, G)$ into a real analytic Kähler manifold $(S, G)$. Then $g$ is real analytic. Let $D_{p}^{g}: U \rightarrow \mathbb{R}$ and $D_{\varphi(p)}^{G}: V \rightarrow \mathbb{R}$ be the diastasis functions of $(M, g)$ and $(S, G)$ around $p$ and $\varphi(p)$ respectively. Then $\varphi^{-1}\left(D_{\varphi(p)}^{G}\right)=D_{p}^{g}$ on $\varphi^{-1}(V) \cap U$.

When $(S, G)$ is the $N$-dimensional complex projective space $S=\mathbb{C} P^{N}$ equipped with with the Fubini-Study metric $G=g_{F S}$, one can show that for all $p \in \mathbb{C} P^{N}$ the diastasis function $D_{p}^{g_{F S}}$ around $p$ is globally defined except in the cut locus $H_{p}$ of $p$ where it blows up. Moreover, $e^{-D_{p}^{g} F S}$ is globally defined (and smooth) on $\mathbb{C} P^{N}$ (see [8] or [25] for details).

Then, by Lemma 4.1 one immediately gets the following:

Lemma 4.2 Let $g$ be a projectively induced Kähler metric on a complex manifold $M$. Then, $e^{-D_{p}^{g}}$ is globally defined on all $M$.

Corollary 4.3 Let $g_{*}$ be the Kähler metric on $\mathbb{C}^{*}$ whose associated Kähler form is given by $\omega_{*}=\frac{i}{2} \partial \bar{\partial}|\eta|, \eta=x+i y$. Then $g_{*}$ is not projectively induced.

Proof: Fix any point $\alpha \in \mathbb{C}^{*}$. A globally defined Kähler potential $\Phi$ for the Kähler metric $g_{*}$ around $\alpha$ is given by $\Phi(\eta)=|\eta|$ and Calabi's diastasis function around $\alpha$ reads as

$$
D_{\alpha}^{g_{*}}: U \rightarrow \mathbb{R}, \eta \mapsto|\eta|+|\alpha|-\sqrt{\eta \bar{\alpha}}-\sqrt{\bar{\eta} \alpha}
$$

where $U \subset \mathbb{C}^{*}$ is suitable simply-connected open subset of $\mathbb{C}^{*}$ around $\alpha$ (as a maximal domain of defintion of $D_{\alpha}^{g_{*}}$ one can take $U=\mathbb{C}^{*} \backslash L$ where $L$ is any half-line starting from the origin of $\mathbb{C}=\mathbb{R}^{2}$ such that $\left.\alpha \notin L\right)$. The function $D_{\alpha}^{g_{*}}$, as well as the function $e^{-D_{\alpha}^{g_{*}}}$, cannot be extended to all $\mathbb{C}^{*}$. Hence we are done by Lemma 4.2 .

We are now in the position to prove that our estimate is sharp.

Theorem 4.4 Let $g$ be the Kähler metric on the Kepler manifold X whose associated Kähler form is given by (2.12). Then $g$ is not projectively induced. 
Proof: First observe that the map

$$
j:\left(\mathbb{C}^{*}, g_{*}\right) \rightarrow(X, g)
$$

defined by $j(z)=(\eta, i \eta, 0, \ldots, 0)$ is a Kähler immersion satisfying $j^{*}(g)=g_{*}$, with $g_{*}$ as in Corollary 4.3. Assume by contradiction that $g$ is projectively induced, namely there exists $N \leq \infty$ and a Kähler immersion $\varphi:(X, g) \rightarrow\left(\mathbb{C} P^{N}, g_{F S}\right)$. Then the map $\varphi \circ j:\left(\mathbb{C}^{*}, g_{*}\right) \rightarrow$ $\left(\mathbb{C} P^{N}, g_{F S}\right)$ would be a Kähler immersion contradicting Corollary 4.3.

\section{Estimates of the logarithmic obstruction term}

The aim of this section is two-folded. First, taking advantage of the homogeneity structure of the Kepler manifold we introduce global polar-angular coordinates. As an outcome, we are able to write down the explicit form of the operator $\partial \bar{\partial}$ in such coordinates. Secondly, we show precise asymptotic expansion and estimates of the logarithmic error term. This is a novel result, as far as we know. A key functional-analytic ingredient of our arguments in the proof of the representation formula in the homogeneous coordinates is the fact that the distortion function for the Kepler manifold $X$ depends only on the rescaled by the factor $\sqrt{2}$ Euclidean distance to the origin in $C^{n+1}$ identified with $\mathbb{R}^{2 n+2}$ by the canonical complex structure $J_{0}$. More precisely, the Kepler manifold is given by the isotropic cone $C$, defined globally by

$$
C=] 0,+\infty[\times \widetilde{C}
$$

where

$$
\widetilde{C}=\left\{e+i s \in \mathbb{C}^{n+1}:(e, s) \in S^{n} \times S^{n}: e \cdot s=0\right\}
$$

is the unitary tangent bundle of $S^{n}$. Here $e=\left(e_{1}, \ldots, e_{n+1}\right), s=\left(s_{1}, \ldots, s_{n+1}\right)$, and $e \cdot s=$

$\sum_{j=1}^{n+1} e_{j} s_{j}$. We can parameterize explicitly $\widetilde{C}$ by using twice the classical angular variables in $\mathbb{R}^{n+1}$, namely, $e=e(\varphi)$ and $s=s(\psi)$, with

$$
\begin{aligned}
e_{1} & =\sin \varphi_{1} \ldots \sin \varphi_{n-1} \sin \varphi_{n}, \\
e_{2} & =\sin \varphi_{1} \ldots \sin \varphi_{n-1} \cos \varphi_{n}, \\
e_{3} & =\sin \varphi_{1} \ldots \sin \varphi_{n-2} \cos \varphi_{n-1}, \\
\ldots & =\ldots \\
e_{n+1} & =\cos \varphi_{1},
\end{aligned}
$$

and

$$
\begin{aligned}
s_{1} & =\sin \psi_{1} \ldots \sin \psi_{n-1} \sin \psi_{n}, \\
s_{2} & =\sin \psi_{1} \ldots \sin \psi_{n-1} \cos \psi_{n}, \\
s_{3} & =\sin \psi_{1} \ldots \sin \psi_{n-2} \cos \psi_{n-1}, \\
\ldots & =\ldots \\
s_{n+1} & =\cos \psi_{1},
\end{aligned}
$$

with $\varphi_{1} \in\left[0,+2 \pi\left[, \psi_{1} \in\left[0,2 \pi\left[, \varphi_{j} \in\left[0, \pi\left[, \psi_{j} \in[0, \pi[, j=2, \ldots, n\right.\right.\right.\right.\right.\right.$. 
Clearly $\widetilde{C} \subset S^{2 n+1}(\sqrt{2})$ since

$$
e+i s \in S^{2 n+1}(\sqrt{2})=\left\{\zeta \in \mathbb{C}^{n+1}: \zeta, \bar{\zeta}=2\right\}, \quad \text { if } e+i s \in \widetilde{C} .
$$

If we define $S^{2 n+1}(r)$ by the angular coordinates in $\mathbb{R}^{2 n+2}=\mathbb{C}^{2 n+2}$ using the coordinates $\zeta=\xi+i \eta \in \mathbb{C}^{n+1}$ and $\left(\xi_{1}, \eta_{1}, \ldots, \xi_{n+1}, \eta_{n+1}\right) \in \mathbb{R}^{2 n+2} \backslash 0$ (identifying with $\zeta=\xi+i \eta \in$ $\left.\mathbb{C}^{n+1} \backslash 0\right)$ by the standard angular cordinates we will have that $S^{2 n+1}(\sqrt{2})$ is defined by $r=\sqrt{2}$, where

$$
\begin{aligned}
\xi_{1} & =r \sin \theta_{1} \ldots \sin \theta_{2 n} \sin \theta_{2 n+1} \\
\eta_{1} & =r \sin \theta_{1} \ldots \sin \theta_{2 n} \cos \theta_{2 n+1} \\
\ldots & =\ldots \\
\xi_{n+1} & =r \sin \theta_{1} \cos \theta_{2} \\
\eta_{n+1} & =r \cos \theta_{1}
\end{aligned}
$$

with $r>0, \theta_{1} \in\left[0,+2 \pi\left[, \theta_{j} \in[0, \pi[, j=2, \ldots, 2 n+1\right.\right.$. However, such coordinates do not provide an easy definition of $\widetilde{C}$ by implicit function theorem.

We construct an embedding $\widetilde{C}$ in $S^{2 n+1}(\sqrt{2})$ compatible with the standard complex structure $J_{0}$ of $\mathbb{C}^{n+1}$ by introducing apparently new angular coordinates on $S^{2 n+1}(\sqrt{2})$ which differ from the traditional ones. More precisely, we set

$$
\begin{aligned}
\xi_{1} & =r \sin \varphi_{1} \ldots \sin \varphi_{n-1} \sin \varphi_{n} \cos \theta \\
\xi_{2} & =r \sin \varphi_{1} \ldots \sin \varphi_{n-1} \cos \varphi_{n} \cos \theta \\
\xi_{3} & =r \sin \varphi_{1} \ldots \sin \varphi_{n-2} \cos \varphi_{n-1} \cos \theta \\
\ldots & =\ldots \\
\xi_{n+1} & =r \cos \varphi_{1} \cos \theta \\
\eta_{1} & =r \sin \psi_{1} \ldots \sin \psi_{n-1} \sin \psi_{n} \sin \theta \\
\eta_{2} & =r \sin \psi_{1} \ldots \sin \psi_{n-1} \cos \psi_{n} \sin \theta \\
\eta_{3} & =r \sin \psi_{1} \ldots \sin \psi_{n-2} \cos \psi_{n-1} \sin \theta \\
\ldots & =\cdots \\
\eta_{n+1} & =r \cos \psi_{1} \sin \theta,
\end{aligned}
$$

where $r>0$, and $\varphi=\left(\varphi_{1}, \ldots, \varphi_{n}\right) \in I, \psi=\left(\psi_{1}, \ldots, \psi_{n}\right) \in J, \theta \in[0,2 \pi[$,

$$
\begin{aligned}
I & =I_{1} \times I_{2} \times \ldots I_{n} \\
J & =J_{1} \times J_{2} \times \ldots J_{n}
\end{aligned}
$$

where $I_{1}, J_{1}$ are semi-closed intervals of length $2 \pi$ while $I_{2}, \ldots, I_{n}, J_{2}, \ldots, J_{n}$ are semi-closed intervals of length $\pi$.

Example 5.1 Let $n=1$. Then the polar coordinates (5.53), (15.54) for $\mathbb{C}^{2}=\mathbb{R}^{4}$ become

$$
\begin{aligned}
& \xi_{1}=r \sin \varphi_{1} \cos \theta \\
& \xi_{2}=r \cos \varphi_{1} \cos \theta
\end{aligned}
$$


and

$$
\begin{aligned}
& \eta_{1}=r \sin \psi_{1} \sin \theta \\
& \eta_{2}=r \cos \psi_{1} \sin \theta .
\end{aligned}
$$

If $n=3$, the polar coordinates (5.53), (5.54) for $\mathbb{C}^{3}=\mathbb{R}^{6}$ become

$$
\begin{aligned}
& \xi_{1}=r \sin \varphi_{1} \sin \varphi_{2} \cos \theta \\
& \xi_{2}=r \sin \varphi_{1} \cos \varphi_{2} \cos \theta \\
& \xi_{3}=r \sin \cos \varphi_{1} \cos \theta
\end{aligned}
$$

and

$$
\begin{aligned}
& \eta_{1}=r \sin \psi_{1} \sin \psi_{2} \sin \theta \\
& \eta_{2}=r \sin \psi_{1} \cos \psi_{2} \sin \theta \\
& \eta_{3}=r \cos \psi_{1} \sin \theta,
\end{aligned}
$$

Remark 5.2 The polar coordinates in (5.53), (5.54) might be viewed as a geometric construction of the odd dimensional sphere $S^{2 n+1}(\mu)$ with radius $\mu>0$ of the following type: we consider the product of the $n$-dimensional spheres $S^{n}\left(2^{-1 / 2} \mu\right)$ in $n+1$-dimensional real subspaces $\operatorname{Im} \zeta=0$ and $\operatorname{Re} \zeta=0$ plus a rotation with the angle $\theta$.

We propose an apparently new representation of the Kepler manifold by means of the co-dimension 2 sub-manifold of $\mathbb{C}^{n+1} \backslash 0$ using the angular variables (5.53), (5.54).

Proposition 5.3 The Kepler manifold $C$ is defined in the polar coordinates $r>0,(\varphi, \psi, \theta)$ in (5.53), (5.54) by

$$
\left\{(r, \varphi, \psi, \theta): \theta=\frac{\pi}{4}, \quad H(\varphi, \psi)=0\right\}
$$

where

$$
\begin{aligned}
H(\varphi, \psi) & :=e(\varphi) \cdot s(\psi) \\
& =\cos \left(\varphi_{n}-\psi_{n}\right) \prod_{j=1}^{n-1}\left(\sin \varphi_{j} \sin \psi_{j}\right)+\widetilde{H}\left(\varphi^{\prime}, \psi^{\prime}\right)
\end{aligned}
$$

with $\varphi^{\prime}=\left(\varphi_{1}, \ldots, \varphi_{n-1}\right), \psi^{\prime}=\left(\psi_{1}, \ldots, \psi_{n-1}\right)$ and

$$
\widetilde{H}\left(\varphi^{\prime}, \psi^{\prime}\right)=\cos \varphi_{1} \cos \psi_{1}+\sum_{j=2}^{n-1} \cos \varphi_{j} \cos \psi_{j} \prod_{\ell=1}^{j-1} \sin \varphi_{\ell} \sin \psi_{\ell}
$$

Clearly, $\widetilde{C}$ is embedded in $S^{2 n+1}(\sqrt{2})$ by the equations

$$
r=\sqrt{2}, \quad \theta=\frac{\pi}{4}, \quad H(\varphi, \psi)=0
$$


and therefore

$$
\rho=|x|=\frac{r}{\sqrt{2}}
$$

Since $\widetilde{C}$ is compact we can find a finite covering $\widetilde{C}=\bigcup_{k=1}^{d} \widetilde{U}_{k}$ of open charts $\widetilde{U}_{k}, k=1, \ldots, d$, which yields

$$
\left.C=\bigcup_{k=1}^{d}\right] 0,+\infty\left[\times \widetilde{U}_{k}\right.
$$

where each $\widetilde{U}_{k}$ is diffeomorphic to an open set $U_{k} \subset \mathbb{R}^{2 n-1}$, with local coordinates $\Theta=\Theta^{k}=$ $\left(\Theta_{1}^{k}, \ldots, \Theta_{2 n-1}^{k}\right) \in U_{k}$. For every $k \in\{1, \ldots d\}$ the two form $\partial \bar{\partial} f, f$ being a smooth function on $C$, can be written in the (cylindric) coordinates $(\rho, \Theta) \in] 0,+\infty\left[\times U_{k}\right.$ as follows

$$
\begin{aligned}
\partial \bar{\partial} f & =\sum_{\ell=1}^{2 n-1} \rho\left(\theta_{0}^{\ell} \partial_{\rho}^{2}+\theta_{1}^{\ell} \rho^{-1} \partial_{\rho} L_{1}^{\ell}+\rho^{-1} b^{\ell} \partial_{\rho}+\rho^{-2} \widetilde{\Delta}_{\ell}\right) f(\rho, \Theta) d \rho \wedge d \Theta_{\ell} \\
& +\sum_{j, \ell=1}^{2 n-1} \rho^{2}\left(\theta_{0}^{j \ell} \partial_{\rho}^{2}+\theta_{1}^{j \ell} \rho^{-1} \partial_{\rho} L_{1}^{j \ell}+\rho^{-1} b^{j \ell} \partial_{\rho}+\rho^{-2} \widetilde{\Delta}^{j \ell}\right) f(\rho, \Theta) d \Theta_{j} \wedge d \Theta_{\ell},
\end{aligned}
$$

where $\theta_{0}^{\ell}, \theta_{0}^{j \ell}, \theta_{1}^{\ell}, \theta_{1}^{j \ell} b^{\ell}, b^{j \ell}$ are real-valued real analytic functions on $U_{j}, L_{1}^{\ell}$, $L_{1}^{j \ell}$ are real tangential vector fields to $\widetilde{C}$ with real analytic coefficients while $\widetilde{\Delta}^{j \ell}, \widetilde{\Delta}^{j \ell}$ are second order linear analytic differential operators without zero order term on $\widetilde{C}\left(i . e ., \Delta^{j \ell} 1=\widetilde{\Delta}^{j \ell} 1=0\right.$ ), $j, \ell=1, \ldots, 2 n-1$. In particular, if $f$ is constant on $\widetilde{C}$, i.e., $f=f(\rho)$, we have

$$
\begin{aligned}
\partial \bar{\partial} f & =\sum_{\ell=1}^{2 n-1}\left(\theta_{0}^{\ell}(\Theta) \rho f^{\prime \prime}(\rho)+b^{\ell}(\Theta) f^{\prime}(\rho)\right) d \rho \wedge d \Theta_{\ell} \\
& +\sum_{j, \ell=1}^{2 n-1}\left(\theta_{0}^{j \ell}(\Theta) \rho^{2} f^{\prime \prime}(\rho)+b^{j \ell}(\Theta) \rho f^{\prime}(\rho)\right) d \Theta_{j} \wedge d \Theta_{\ell} .
\end{aligned}
$$

Proof: We start by recalling the representation of $\partial \bar{\partial} f$ in $\mathbb{C}^{n+1}$ by identifying $\mathbb{C}^{n+1}=$ $\mathbb{R}^{2 n+2}$ by the canonical complex structure $J_{0}$ for $n \geq 1$. Recall that if $n=0$ we have

$$
\partial \bar{\partial} f=2 i\left(f_{x x}+f_{y y}\right) d x \wedge d y
$$

with $z=x+y i \in \mathbb{C}$.

Lemma 5.1 Let $n \geq 1$ and $f \in C^{\infty}\left(\mathbb{C}^{n+1} \backslash 0\right)=C^{\infty}\left(\mathbb{R}^{2 n+2} \backslash 0\right)$ with coordinates $\zeta=\xi+i \eta$. Then we can write $\partial \bar{\partial} f$ in the standard polar coordinates in $\mathbb{R}^{2 n+2} \backslash 0$ :

$$
\begin{gathered}
\partial \bar{\partial} f=\frac{i}{2} \sum_{\ell=1}^{n+1} \partial_{\zeta_{\ell}} \partial_{\bar{\zeta}_{\ell}} f d \zeta_{\ell} \wedge d \bar{\zeta}_{\ell} \\
=\sum_{\ell=1}^{n+1}\left(f_{\xi_{\ell} \xi_{\ell}}+f_{\eta_{\ell} \eta_{\ell}}\right) d \xi_{\ell} \wedge d \eta_{\ell}
\end{gathered}
$$


Moreover, in the the standard polar coordinates in $\mathbb{R}^{2 n+2} \backslash 0$ defined by (15.52) we can write

$$
\partial \bar{\partial} f=\rho \sum_{\ell=1}^{2 n+1} \Omega^{\ell}[f] d \rho \wedge d \Theta_{\ell}+\rho^{2} \sum_{j, \ell=1}^{2 n+1} \Omega^{j \ell}[f] d \Theta_{j} \wedge d \Theta_{\ell}
$$

with

$$
\begin{aligned}
\Omega^{\ell}[f] & =E_{0}^{\ell}(\Theta) \partial_{\rho}^{2} f+E_{1}^{\ell}(\Theta) \rho^{-1} \partial_{\rho} L_{1}^{\ell ; E}\left(\Theta, \partial_{\Theta}\right) f \\
& +\rho^{-1} e^{\ell}(\Theta) \partial_{\rho} f+\rho^{-2} \widetilde{\Delta}_{\ell}^{E}\left(\Theta, \partial_{\Theta}\right) f \\
\Omega^{j \ell}[f] & =E_{0}^{j \ell}(\Theta) \partial_{\rho}^{2} f+E_{1}^{j \ell}(\Theta) \rho^{-1} \partial_{\rho} L_{1}^{j \ell ; E}\left(\Theta, \partial_{\Theta}\right) f \\
& +\rho^{-1} e^{j \ell}(\Theta) \partial_{\rho} f+\rho^{-2} \widetilde{\Delta}^{j \ell ; E}\left(\Theta, \partial_{\Theta}\right) f
\end{aligned}
$$

where $E_{0}^{\ell}, E_{0}^{j \ell}, E_{1}^{\ell}, E_{1}^{j \ell} e^{\ell}$, e $e^{j \ell}$ are real-valued real analytic functions of $\theta, L_{1}^{\ell ; E}, L_{1}^{j \ell ; E}$ are real tangential vector fields to $S^{2 n+1}$ with real analytic coefficients while $\widetilde{\Delta}^{j \ell ; E}, \widetilde{\Delta}^{j \ell ; E}$ are second order linear analytic differential operators without zero order term on $S^{2 n+1}$ (i.e., $\left.\Delta^{j \ell ; E}(1)=\widetilde{\Delta}^{j \ell ; E}(1)=0\right), j, \ell=1, \ldots, 2 n+1$.

Proof. The first identity (5.68) is immediate. Next, by the standard calculus on two forms and change of the variables, we obtain

$$
\begin{aligned}
\partial \bar{\partial} f & =\sum_{\ell=1}^{n+1}\left(f_{\xi_{\ell} \xi_{\ell}}+f_{\eta_{\ell} \eta_{\ell}}\right) d \xi_{\ell} \wedge d \eta_{\ell} \\
& =\sum_{j=1}^{n+1}\left(f_{\xi_{j} \xi_{j}}+f_{\eta_{j} \eta_{j}}\right) \sum_{\ell=1}^{2 n+1}\left(\partial_{\rho} \xi_{j} \partial_{\Theta_{\ell}} \eta_{j}-\partial_{\rho} \eta_{j} \partial_{\Theta_{\ell}} \xi_{j}\right) d \rho \wedge d \Theta_{\ell} \\
& +\sum_{j=1}^{n+1}\left(f_{\xi_{j} \xi_{j}}+f_{\eta_{j} \eta_{j}}\right) \sum_{k, \ell=1}^{2 n+1}\left(\partial_{\Theta_{k}} \xi_{j} \partial_{\Theta_{\ell}} \eta_{j}-\partial_{\Theta_{k}} \eta_{j} \partial_{\Theta_{\ell}} \xi_{j}\right) d \Theta_{k} \wedge d \Theta_{\ell} \\
& =\sum_{\ell=1}^{2 n+1}\left(\sum_{j=1}^{n+1}\left(f_{\xi_{j} \xi_{j}}+f_{\eta_{j} \eta_{j}}\right)\left(\partial_{\rho} \xi_{j} \partial_{\Theta_{\ell}} \eta_{j}-\partial_{\rho} \eta_{j} \partial_{\Theta_{\ell}} \xi_{j}\right)\right) d \rho \wedge d \Theta_{\ell} \\
& +\sum_{k, \ell=1}^{2 n+1}\left(\sum_{j=1}^{n+1}\left(f_{\xi_{j} \xi_{j}}+f_{\eta_{j} \eta_{j}}\right)\left(\partial_{\Theta_{k}} \xi_{j} \partial_{\Theta_{\ell}} \eta_{j}-\partial_{\Theta_{k}} \eta_{j} \partial_{\Theta_{\ell}} \xi_{j}\right)\right) d \Theta_{k} \wedge d \Theta_{\ell} .
\end{aligned}
$$

We conclude by writing $f_{\xi_{j} \xi_{j}}+f_{\eta_{j} \eta_{j}}$ in the polar coordinates $(\rho, \Theta)$.

Set $\Phi=(\varphi, \psi) \in I \times J$, where $I$ (respectively $J$ ) is as in (5.55) (respectively, (5.56) ). We can apply the implicit function theorem in the global coordinates on $\widetilde{C}$ is applicable outside the singular set $\widetilde{C}_{S}(I \times J)$ (in the fixed angular variables) defined by the system

$$
H(\Phi)=0, \quad d_{\Phi} H(\Phi)=0 .
$$

In fact, choose $V \subset \subset I \times J \backslash \widetilde{C}_{S}(I \times J)$, then by the implicit function theorem, there exists $s \in\{1, \ldots, n\}$ such that $H(\Phi)=0$ on $V$ defines $\varphi_{s}=\varphi_{s}\left(\varphi^{s}, \psi\right)$ or $\psi_{s}=\psi_{s}\left(\varphi, \psi^{s}\right)$, where

$$
\varphi^{s}=\left(\varphi_{1}, \ldots, \varphi_{s-1}, \varphi_{s+1}, \ldots, \varphi_{n}\right), \quad \varphi^{s}=\left(\varphi_{1}, \ldots, \varphi_{s-1}, \varphi_{s+1}, \ldots, \varphi_{n}\right) .
$$


with $\Theta=\left(\varphi^{s}, \psi\right)$ or $\Theta=\left(\varphi, \psi^{s}\right)$ belonging to some open $V^{\prime} \subset \mathbb{R}^{2 n-1}$. Clearly $V^{\prime}$ defines a chart and in view of the compactness of $S^{2 n+1}(r)$ we can choose a finite number by varying $I$ and $J$ in the definitions (5.55), (5.56).

It is well known by the calculus on manifolds that

$$
\begin{aligned}
\partial_{z_{j}} \partial_{\overline{z_{\ell}}} f & =\sum_{r=1}^{2 n-1}\left(\Omega_{r}^{j \ell}(\Theta) \partial_{\rho}^{2}+\rho^{-1} \partial_{\rho} T_{r}^{j \ell}+\rho^{-1} \tilde{b}_{r}^{j \ell} \partial_{\rho}+\rho^{-2} D_{r}^{j \ell}\right) f \\
& +\sum_{r, s=1}^{2 n-1}\left(\Omega_{r s}^{j \ell} \partial_{\rho}^{2}+\rho^{-1} \partial_{\rho} T_{r s}^{j \ell}+\rho^{-1} \tilde{b}_{r s}^{j \ell} \partial_{\rho}+\rho^{-2} D_{r s}^{j \ell}\right) f
\end{aligned}
$$

and

$$
\begin{aligned}
d z_{j} \wedge d \bar{z}_{\ell} & =\rho \sum_{p=1}^{2 n-1} \Gamma_{p}^{j \ell}(\Theta) d \rho \wedge d \Theta_{p} \\
& +\rho^{2} \sum_{p, q=1}^{2 n-1} \Gamma_{p q}^{j \ell}(\Theta) d \Theta_{p} \wedge d \Theta_{q}
\end{aligned}
$$

where

$$
\begin{aligned}
& T_{r}^{j \ell}=\sum_{q=1}^{2 n-1} T_{r ; q}^{j \ell}(\Theta) \partial_{\Theta_{q}} \\
& T_{r s}^{j \ell}=\sum_{q=1}^{2 n-1} T_{r s ; q}^{j \ell}(\Theta) \partial_{\Theta_{q}}
\end{aligned}
$$

are tangential vector field to $\widetilde{C} \cap \tilde{U}_{j}$ with real analytic coefficients while

$$
\begin{aligned}
D_{r}^{j \ell} & =\sum_{p, q=1}^{2 n-1} D_{r ; p q}^{j \ell}(\Theta) \partial_{\Theta_{p}} \partial_{\Theta_{q}} \\
& \left.+\sum_{q=1}^{2 n-1} H_{r ; q}^{j \ell}(\Theta)\right) \partial_{\Theta_{q}} \\
D_{r s}^{j \ell} & =\sum_{p, q=1}^{2 n-1} D_{r s ; p q}^{j \ell}(\Theta) \partial_{\Theta_{p}} \partial_{\Theta_{q}} \\
& +\sum_{q=1}^{2 n-1} H_{r s ; q}^{j \ell}(\Theta) \partial_{\Theta_{q}}
\end{aligned}
$$

are second order linear analytic differential operator on $\widetilde{C} \cap \tilde{U}_{j}$ without zero order terms. Clearly, (5.80) and (5.74) yield (5.66). The proof is complete.

We observe that the definition of $T_{m}(z)$ implies that

$$
\begin{aligned}
\log T_{m}(z) & =n \log (2 m)+\log \left(\frac{T_{m}(z)}{2 m^{n}}\right) \\
& =n \log (2 m)+\log F(m|x|) \\
& =n \log (2 m)+\log \left(\sum_{j=0}^{n-2} \frac{a_{j}}{(m|x|)^{j}}+\Phi(m|x|)+\Psi(m|x|)\right) .
\end{aligned}
$$


Set

$$
F(\rho)=\sum_{j=0}^{n-2} \frac{a_{j}}{\rho^{j}}+\Phi(\rho)+\Psi(\rho) .
$$

Clearly we can rewrite $(\underline{5.80})$ on $C=] 0,+\infty[\times \tilde{C}$ as follows:

$$
\log T_{m}(z)=n \log (2 m)+\log F(m|x|)=n \log (2 m)+\log F(m \rho) .
$$

We note that (5.80) implies for every fixed $\rho>0$ the function $\log T_{m}(z)$ is constant $C=$ $\{\rho\} \times \tilde{C}$. The representaion formula (5.82) yileds

$$
\left.\mathcal{E}_{m}\right|_{C}=\frac{i}{2 m} \partial \bar{\partial}\left(\operatorname { l o g } \left(\left.F(m|x|)\right|_{C}\right.\right.
$$

We show the main result of the present section.

Theorem 5.4 There exist $2(n+1)^{2}$ real analytic functions $\sigma_{k \ell}(\Theta), \tau_{k \ell}(\Theta), \overline{\sigma_{k \ell}}=\sigma_{\ell k}, \overline{\tau_{k \ell}}=$ $\sigma_{\ell k}, k, \ell=1, \ldots, n+1$, defined on $\widetilde{C}$ such the 2 -form the obstruction term

$$
\begin{aligned}
\left.\mathcal{E}_{m}\right|_{C_{j}}(\rho, \Phi) & :=\left.\mathcal{E}_{m}\right|_{] 0,+\infty\left[\times\left(\tilde{C} \cap U_{j}\right.\right.}(\rho, \Phi) \\
& =\sum_{q=1}^{2 n-1} \mathcal{E}_{m}^{q}(\rho, \Theta) d \rho \wedge d \Theta_{q} \\
& +\sum_{p, q=1}^{2 n-1} \mathcal{E}_{m}^{p q}(\rho, \Theta) d \Theta_{p} \wedge d \Theta_{q}
\end{aligned}
$$

where

$$
\begin{aligned}
\mathcal{E}_{m}^{q}(\rho, \Theta) & =\theta_{0}^{q}(\Theta) m \rho \frac{F^{\prime \prime}(m \rho) F(m \rho)-\left(F^{\prime}(m \rho)\right)^{2}}{F^{2}(m \rho)}+b_{q}(\Theta) \frac{F^{\prime}(m \rho)}{F(m \rho)} \\
& =\frac{1}{m^{2} \rho^{2}}\left(\theta_{0}^{q}(\Theta) \sum_{s=0}^{N} \frac{p_{s}}{\rho^{s} m^{s}}+b_{q}(\Theta) \sum_{s=0}^{N} \frac{q_{s}}{\rho^{s} m^{s}}\right) \\
& +\theta_{0}^{q}(\Theta) R_{N}^{1}(m \rho)+b_{q}(\Theta) R_{N}^{2}(m \rho) \\
\mathcal{E}_{m}^{p q}(\rho, \Theta) & =\theta_{0}^{p q}(\Theta) m \rho^{2} \frac{F^{\prime \prime}(m \rho) F(m \rho)-\left(F^{\prime}(m \rho)\right)^{2}}{F^{2}(m \rho)}+b_{p q}(\Theta) \rho \frac{F^{\prime}(m \rho)}{F(m \rho)} \\
& =\frac{1}{m^{2} \rho}\left(\theta_{0}^{p q}(\Theta) \sum_{s=0}^{N} \frac{p_{s}}{\rho^{s} m^{s}}+b_{p q}(\Theta) \sum_{s=0}^{N} \frac{p_{s}}{\rho^{s} m^{s}}\right) \\
& +\theta_{0}^{p q}(\Theta) \rho R_{N}^{1}(m \rho)+b_{p q}(\Theta) \rho R_{N}^{2}(m \rho)
\end{aligned}
$$

for all $N \in \mathbb{N}$, where the real constants $p_{s}, q_{s}$ depend on $a_{1}, \ldots, a_{n-2}$ by explicit formulas while $\theta_{0}^{q}(\Theta), b_{q}(\Theta), \theta_{0}^{p q}(\Theta), b_{p q}(\Theta)$ are real-valued real analytic functions on $\tilde{C}$. The reminders $R_{N}^{\mu}$, $\mu=1,2$ satisfy the following estimates: for every $\delta_{0}>0$ one can find positive constants $A_{\mu}$, $B_{\mu}, \mu=1,2$, such that

$$
\left|\partial_{x}^{\alpha}\left(R_{N}^{\mu}(m|x|)\right)\right| \leq A_{\mu}^{N+1} B_{\mu}^{|\alpha|} N ! \alpha ! m^{-N-1}|x|^{-N-|\alpha|-3}
$$


for all $N \in \mathbb{Z}_{+}, \alpha \in \mathbb{Z}_{+}^{n},|x| \geq \delta_{0}, m \geq 1$. In particular, there exist positive constants $c_{0}, \mu_{0}, A_{0}$ such that if we choose $N=N(m)=1+\left[e^{c_{0} m}\right]$ then ${\widetilde{R^{\mu}}}_{m}(m \rho):=R_{N(m)}^{\mu}(m \rho)$ are exponentially small in the following sense:

$$
\left|\partial_{x}^{\alpha}\left(\widetilde{R}_{m}(m|x|)\right)\right| \leq A_{0} B_{\mu}^{|\alpha|} \alpha ! e^{-c_{0} m|x|},
$$

for all $\alpha \in \mathbb{Z}_{+}^{n},|x| \geq \delta_{0}, m \geq 1$. Finally, we can summarize the asymptotic estimates above as follows: every $N \in \mathbb{N}, \delta>0$ one can find $C>0$ such that

$$
\begin{aligned}
\mathcal{E}_{m}^{q}(\rho, \Theta) & =\frac{1}{\rho^{2} m^{2}} \sum_{s=1}^{N} \frac{\varkappa_{s}^{q}(\Theta)}{m^{s} \rho^{s}}+R_{N}^{q}(m \rho, \Theta) \\
\mathcal{E}_{m}^{p q}(\rho, \Theta) & =\frac{1}{\rho m^{2}} \sum_{s=1}^{N} \frac{\varkappa_{s}^{p q}(\Theta)}{m^{s} \rho^{s}}+\rho R_{N}^{p q}(m \rho, \Theta),
\end{aligned}
$$

where

$$
\begin{aligned}
& \left|\partial_{\rho}^{\beta} \partial_{\Theta}^{\alpha}\left(R_{N}^{q}(\rho, \Theta ; m)\right)\right| \leq C^{N+1+|\alpha|} N ! \alpha ! m^{-N-3} \rho^{-N-3-\beta} \\
& \left|\partial_{\rho}^{\beta} \partial_{\Theta}^{\alpha}\left(R_{N}^{p q}(\rho, \Theta ; m)\right)\right| \leq C^{N+1+|\alpha|} N ! \alpha ! m^{-N-3} \rho^{-N-2-\beta}
\end{aligned}
$$

for $m \geq 1,|x| \geq \delta, \beta \in \mathbb{Z}_{+}, \alpha \in \mathbb{Z}_{+}^{2 n-1}, k, \ell=1, \ldots, n+1, p, q=1, \ldots 2 n-1$.

Proof. Fix $m \in N$. The first step of the proof consists in applying (5.67) for

$$
f(\rho)=\frac{i}{2 m} \log (F(m \rho)) .
$$

We note that by (5.67) for $f(\rho)=\frac{i}{2 m} \log (F(m \rho))$ and the identities

$$
\begin{aligned}
\partial_{\rho}(\log (F(m \rho)) & =m \frac{F^{\prime}(m \rho)}{F(m \rho)} \\
\partial_{\rho}^{2}(\log (F(m \rho)) & =m^{2} \frac{F^{\prime \prime}(m \rho) F(m \rho)-\left(F^{\prime}(m \rho)\right)^{2}}{F^{2}(m \rho)}
\end{aligned}
$$

we get

$$
\begin{aligned}
\mathcal{E}_{m}^{q}(\rho, \Theta) & =\frac{i}{2} \frac{\Gamma^{q}(\Theta, m \rho)}{F^{2}(m \rho)} \\
\mathcal{E}_{m}^{p q}(\rho, \Theta) & =\frac{i m \rho}{2} \frac{\Gamma^{p q}(\Theta, m \rho)}{F^{2}(m \rho)}
\end{aligned}
$$

where

$$
\begin{aligned}
\Gamma^{q}(\Theta, y) & =\theta_{0}^{q}(\Theta) y\left(F^{\prime \prime}(y) F(y)-\left(F^{\prime}(y)\right)^{2}\right)+b^{q}(\Theta) F^{\prime}(y) F(y) \\
\Gamma^{p q}(\Theta, y) & =\theta_{0}^{p q}(\Theta) y\left(F^{\prime \prime}(y) F(y)-\left(F^{\prime}(y)\right)^{2}\right)+b^{p q}(\Theta) F^{\prime}(y) F(y) .
\end{aligned}
$$

By the asymptotic expansion for $F$ we have

$$
\begin{aligned}
& F^{\prime}(y)=-\sum_{j=1}^{n-2} \frac{j b_{j}}{y^{j+1}}+\Phi^{\prime}(y)+\Psi^{\prime}(y) \\
& F^{\prime \prime}(y)=\sum_{j=1}^{n-2} \frac{j(j+1) b_{j}}{y^{j+2}}+\Phi^{\prime \prime}(y)+\Psi^{\prime \prime}(y)
\end{aligned}
$$


Straightforward calculations of $F^{\prime \prime}(y) F(y),\left(F^{\prime}(y)\right)^{2}, F^{\prime}(y) F(y) / y$ and (5.97) lead to

$$
\begin{aligned}
\Gamma^{q}(\Theta, y) & =\theta_{0}^{q}(\Theta) y\left(F^{\prime \prime}(y) F(y)-\left(F^{\prime}(y)\right)^{2}\right)+b^{q}(\Theta) F^{\prime}(y) F(y), \\
\Gamma^{p q}(\Theta, y) & =\theta_{0}^{p q}(\Theta) y\left(F^{\prime \prime}(y) F(y)-\left(F^{\prime}(y)\right)^{2}\right)+b^{p q}(\Theta) F^{\prime}(y) F(y) .
\end{aligned}
$$

$$
\begin{aligned}
\Gamma^{q}(\Theta, y) & =\sum_{j=1}^{2 n-4} \frac{\mu_{j ; 0}^{q}(\Theta)}{y^{j+2}}+\sum_{j=1}^{2 n-4} \frac{\nu_{j ; 0}^{q}(\Theta)}{y^{j+2}} \\
& +\theta_{0}^{q}(\Theta) E_{1}(y)+b^{q}(\Theta) E_{2}(y) \\
\Gamma^{p q}(\Theta, y) & =\sum_{j=1}^{2 n-4} \frac{\mu_{j ; 0}^{p q}(\Theta)}{y^{j+2}}+\sum_{j=1}^{2 n-4} \frac{\nu_{j ; 0}^{p q}(\Theta)}{y^{j+2}} \\
& +\theta_{0}^{p q}(\Theta) E_{1}(y)+b^{p q}(\Theta)(\Theta) E_{2}(y)
\end{aligned}
$$

where

$$
\begin{aligned}
\mu_{1 ; 0}^{q}(\Theta) & =\theta_{0}^{q}(\Theta) b_{1} b_{0} \\
\mu_{j ; 0}^{q}(\Theta) & =\theta_{0}^{q}(\Theta)\left(b_{0} b_{j}+\sum_{\ell=1}^{j-1} \ell(\ell+1) b_{\ell} b_{j-\ell}-\sum_{\ell=1}^{j-1} \ell(j-\ell) b_{\ell} b_{j-\ell}\right) \\
& =\theta_{0}^{q}(\Theta)\left(j(j+1) b_{0} b_{j}+\sum_{\ell=1}^{j-1} \ell(2 \ell+1-j) b_{\ell} b_{j-\ell}\right) \\
\nu_{1 ; 0}^{q}(\Theta) & =-b^{q}(\Theta) b_{1} b_{0} \\
\nu_{j ; 0}^{q}(\Theta) & =-\theta_{0}^{q}(\Theta)\left(j b_{0} b_{j}+\sum_{\ell=1}^{j-1} \ell b_{\ell} b_{j-\ell}\right) \\
\mu_{1 ; 0}^{p q}(\Theta) & =\theta_{0}^{q}(\Theta) b_{1} b_{0} \\
\mu_{j ; 0}^{p q}(\Theta) & =\theta_{0}^{p q}(\Theta)\left(b_{0} b_{j}+\sum_{\ell=1}^{j-1} \ell(\ell+1) b_{\ell} b_{j-\ell}-\sum_{\ell=1}^{j-1} \ell(j-\ell) b_{\ell} b_{j-\ell}\right) \\
& =\theta_{0}^{p q}(\Theta)\left(j(j+1) b_{0} b_{j}+\sum_{\ell=1}^{j-1} \ell(2 \ell+1-j) b_{\ell} b_{j-\ell}\right) \\
\nu_{1 ; 0}^{p q}(\Theta) & =-b^{p q}(\Theta) b_{1} b_{0} \\
\nu_{j ; 0}^{p q}(\Theta) & =-\theta_{0}^{p q}(\Theta)\left(j b_{0} b_{j}+\sum_{\ell=1}^{j-1} \ell b_{\ell} b_{j-\ell}\right)
\end{aligned}
$$


for $j=2, \ldots, 2 n-4$ and

$$
\begin{aligned}
E_{1}(y) & =(\Phi(y)+\Psi(y)) \sum_{j=1}^{n-2} \frac{j(j+1) b_{j}}{y^{j+2}}+\left(\Phi^{\prime \prime}(y)+\Psi^{\prime \prime}(y)\right) \sum_{j=0}^{n-2} \frac{b_{j}}{y^{j}} \\
& -2\left(\Phi^{\prime}(y)+\Psi^{\prime}(y)\right) \sum_{j=1}^{n-2} \frac{j b_{j}}{y^{j+1}} \\
& +(\Phi(y)+\Psi(y))\left(\Phi^{\prime \prime}(y)+\Psi^{\prime \prime}(y)\right) \\
E_{2}(y) & =-(\Phi(y)+\Psi(y)) \sum_{j=1}^{n-2} \frac{j b_{j}}{y^{j+2}}-\left(\Phi^{\prime}(y)+\Psi^{\prime}(y)\right) \sum_{j=0}^{n-2} \frac{b_{j}}{y^{j+1}} \\
& -(\Phi(y)+\Psi(y))\left(\Phi^{\prime}(y)+\Psi^{\prime}(y)\right)
\end{aligned}
$$

Next, we show an auxiliary assertion.

Lemma 5.2 There exists $K>0$ such that the function $G(y):=F^{-2}(y)$ is uniformly analytic function for $y \geq 2$ satisfying

$$
\frac{1}{F^{2}(y)}=\frac{1}{b_{0}^{2}}+\sum_{j=1}^{\infty} \frac{\beta_{j}}{y^{j}}
$$

where $\beta_{j} \in \mathbb{R}, j=1,2, \ldots$ are defined recursively

$$
\beta_{j}=-2 \frac{b_{j}}{b_{0}}+\widetilde{\beta}_{j}\left(b_{0}, \ldots, b_{j-1}\right), \quad j=1,2, \ldots
$$

and satisfy

$$
\limsup _{j \geq 1} \sqrt[j]{\left|\beta_{j}\right|}<\frac{1}{K}
$$

Moreover,

$$
\frac{1}{F^{2}(m|x|)}=\frac{1}{b_{0}^{2}}+\sum_{j=1}^{\infty} \frac{\beta_{j}}{(m|x|)^{j}}
$$

is uniformly analytic for $|x| \geq K$, uniformly with respect to $m \geq 1$ and the remainder

$$
\widetilde{E}_{N}(y)=\sum_{j=N}^{\infty} \frac{\beta_{j}}{y^{j}}
$$

satisfies, for some positive numbers $\widetilde{A}_{0}, \widetilde{B}_{0}, \widetilde{A}, \widetilde{B}$, the following analytic-Gevrey combinatorial estimates

$$
\left|\left(\frac{d}{d y}\right)^{k}\left(\widetilde{E_{N}}(y)\right)\right| \leq \widetilde{A}_{0}^{N+1} \widetilde{B}_{0}^{k} N ! k ! y^{-N-k-1}
$$

for $y \geq K, k, N \in \mathbb{Z}_{+}$, and

$$
\left|\partial_{x}^{\alpha}\left(\widetilde{E_{N}}(m|x|)\right)\right| \leq \widetilde{A}^{N+1} \widetilde{B}^{\alpha} N ! \alpha ! m^{-N-1}|x|^{-N-\alpha-1}
$$


for all $\alpha \in \mathbb{Z}_{+}^{n}, N \in \mathbb{Z}_{+},|x| \geq K, m \geq 1$.

Proof. We recall that

$$
\frac{1}{(1+\tau)^{2}}=-\frac{d}{d \tau}\left(\frac{1}{1+\tau}\right)=\sum_{j=1}^{\infty}(-1)^{j} j \tau^{j-1}
$$

provided $|\tau|<1$. Set

$$
\varkappa(y)=\frac{1}{b_{0}} \sum_{j=1}^{n-2} \frac{b_{j}}{y^{j}}+\frac{1}{b_{0}} \Phi(y)+\frac{1}{b_{0}} \Psi(y) .
$$

Clearly for every $\varepsilon \in] 0,1\left[\right.$ we can find $K=K_{\varepsilon}>0$ such that

$$
\sup _{y \geq K}|\varkappa(y)|<\varepsilon .
$$

Therefore, by (5.123) and (5.124) we readily obtain the following representation by means of convergent Neumann series

$$
\begin{aligned}
\frac{1}{F^{2}(y)} & =\frac{1}{b_{0}^{2}} \frac{1}{(1+\varkappa(y))^{2}} \\
& =\frac{1}{b_{0}^{2}}+\sum_{j=1}^{\infty} \frac{(-1)^{j+1}(j+1)}{b_{0}^{2}}(\varkappa(y))^{j} .
\end{aligned}
$$

Hence,

$$
\beta_{j}=\left.\frac{1}{j !}\left(\frac{d}{d y}\right)^{j}\left(\sum_{s=1}^{\infty} \frac{(-1)^{s+1}(s+1)}{b_{0}^{2}}(\varkappa(y))^{j}\right)\right|_{y=0} .
$$

We complete the proof of the lemma by plugging (5.123) in (5.125) and applying uniform analytic estimates as in [10, [1].

Next, by the representation formulas derived above, we obtain that

$$
\begin{aligned}
\frac{\Gamma^{q}(\Theta, y)}{F^{2}(y)} & =\sum_{j=1}^{N} \frac{H_{j}^{q}(\Theta, y)}{y^{j+2}}+\sum_{j=1}^{N} \frac{G_{j}^{q}(\Theta, y}{y^{j+2}} \\
& +\theta_{0}^{q}(\Theta) \widetilde{R_{N}^{1}}(\Theta, y)+b^{q}(\Theta) \widetilde{R_{N}^{2}}(y) \\
\frac{\Gamma^{p q}(\Theta, y)}{F^{2}(y)} & =\sum_{j=1}^{N} \frac{H_{j}^{p q}(\Theta, y)}{y^{j+2}}+\sum_{j=1}^{N} \frac{G_{j}^{p q}(\Theta, y}{y^{j+2}} \\
& +\theta_{0}^{p q}(\Theta) \widetilde{R_{N}^{1}}(\Theta, y)+b^{p q}(\Theta) \widetilde{R_{N}^{2}}(y)
\end{aligned}
$$


where

$$
\begin{aligned}
H_{j}^{q}(\Theta, y) & =\theta_{0}^{q}(\Theta)\left(\frac{\mu_{j}}{b_{0}^{2}}+\sum_{\ell=1}^{\min \{2 n-4, j-2\}} \mu_{\ell} \beta_{j-\ell}\right) \\
H_{j}^{p q}(\Theta, y) & =\theta_{0}^{p q}(\Theta)\left(\frac{\mu_{j}}{b_{0}^{2}}+\sum_{\ell=1}^{\min \{2 n-4, j-2\}} \mu_{\ell} \beta_{j-\ell}\right) \\
G_{j}^{q}(\Theta, y) & =\theta_{0}^{q}(\Theta)\left(\frac{\nu_{j}}{b_{0}^{2}}+\sum_{\ell=1}^{\min \{2 n-4, j-1\}} \nu_{\ell} \beta_{j-\ell}\right) \\
G_{j}^{p q}(\Theta, y) & =\theta_{0}^{p q}(\Theta)\left(\frac{\nu_{j}}{b_{0}^{2}}+\sum_{\ell=1}^{\min \{2 n-4, j-1\}} \nu_{\ell} \beta_{j-\ell}\right)
\end{aligned}
$$

and

$$
\begin{aligned}
\widetilde{R_{N}^{1}}(y) & =\sum_{\ell=1}^{2 n-4} \sum_{N+1-\ell \leq j \leq N} \frac{\mu_{\ell} \beta_{j}}{y^{\ell+j+2}} \\
& +\frac{E_{1}(y)}{F^{2}(y)}+\widetilde{E_{N}}(y) \sum_{j=1}^{2 n-4} \frac{\mu_{j}}{y^{j+2}} \\
\widetilde{R_{N}^{2}}(y) & =\sum_{\ell=1}^{2 n-4} \sum_{N+1-\ell \leq j \leq N} \frac{\nu_{\ell} \beta_{j}}{y^{\ell+j+2}} \\
& +\frac{E_{2}(y)}{F^{2}(y)}+\widetilde{E_{N}}(y) \sum_{j=1}^{2 n-4} \frac{\nu_{j}}{y^{j+2}}
\end{aligned}
$$

We conclude the proof of the estimates by straightforward applications of the functionalanalytic arguments in [10], [11] for showing simultaneosly uniform holomorphic extensions and exponential decay on infinity.

Remark 5.5 We point out that (5.88) implies that for a given $m \gg 1$, the optimla choice of $N=N(m)$ for the truncated asymptotic expansion is given by

$$
\sigma(\Phi) \sum_{j=1}^{N(m)} \frac{p_{j}}{|x|^{j+2}} m^{-j}+\tau(\Phi) \sum_{j=1}^{N(m)} \frac{q_{j}}{|x|^{j+2}} m^{-j}
$$

since the remainder is exponentially small for $m \rightarrow+\infty$. We note that similar uniform exponential decay estimates are shown in the framework of analytic-Gevrey pseudodifferential operators, e.g., cf. [36], [10], [11] and the references therein.

Acknowledgements. The authors thank Cornelis Van Der Mee for his precious comments and discussions on various aspects in Quantum Mechancis. Thanks are also due to Ivailo Mladenov and Vassil Tsanov for useful discussions and providing references related to the subject of the paper. 


\section{References}

[1] S. T. Ali and M Engliš, Quantization methods: a guide for physicists and analysts. Rev. Math. Phys. 17 (2005), no. 4, 391-490.

[2] C. Arezzo and A. Loi, Quantization of Kähler manifolds and the asymptotic expansion of Tian-Yau-Zelditch, J. Geom. Phys. 47 (2003), 87-99.

[3] C. Arezzo and A. Loi, Moment maps, scalar curvature and quantization of Kähler manifolds, Comm. Math. Phys. 246 (2004), 543-549.

[4] M. F. Atiyah and R. Bott, The moment map and equivariant cohomology, Topology, 23:1 (1984), 1-28.

[5] F. A. Berezin, Quantization, Izv. Akad. Nauk SSSR Ser. Mat. 38 (1974), 1116-1175 (Russian).

[6] F. A. Berezin, Quantization in complex symmetric spaces, Izv. Akad. Nauk SSSR Ser. Mat. 39 (1975), no. 2, 363-402, 472 (Russian).

[7] F. A. Berezin and M. A. Shubin, The Schrödinger equation. Translated from the 1983 Russian edition by Yu. Rajabov, D. A. Leĭtes and N. A. Sakharova and revised by Shubin. With contributions by G. L. Litvinov and Leĭtes. Mathematics and its Applications (Soviet Series), 66. Kluwer Academic Publishers Group, Dordrecht, 1991. xviii+555 pp.

[8] E. Calabi, Isometric Imbeddings of Complex Manifolds, Ann. of Math. 58 (1953), 1-23.

[9] M. Cahen, S. Gutt, J. H. Rawnsley, Quantization of Kähler manifolds I: Geometric interpretation of Berezin's quantization, J. Geom. Phys. 7 (1990), 45-62.

[10] M. Cappiello, T. Gramchev and L. Rodino, Super-exponential decay and holomorphic extensions for semilinear equations with polynomial coefficients, J. Func. Anal. 237 (2006), 634-654.

[11] M. Cappiello, T. Gramchev and L. Rodino, Gelfand-Shilov spaces, pseudo-differential operators and localization operators, Operator Theory: Advances andApplications, Vol. 172, 297-312, Birkah'auser Verlag, Basel, 2006.

[12] T. Carletti, Exponentially long time stability for non-linearizable analytic germs of $\left(\mathbb{C}^{n}, 0\right)$, Ann Inst. Fourier (Grenoble) 54 (2004), 989-1004.

[13] S. Donaldson, Scalar Curvature and Projective Embeddings, I, J. Diff. Geometry 59 (2001), 479-522.

[14] J. J. Duistermaat and G. J. Heckman, On the variation in the cohomology of the symplectic form of the reduced phase space, Invent. Math. 69:2 (1982), 259-268.

[15] J. J. Duistermaat and G. J. Heckman, Addendum to: "On the variation in the cohomology of the symplectic form of the reduced phase space", Invent. Math. 72:1 (1983), 153-158.

[16] M. Engliš, Berezin Quantization and Reproducing Kernels on Complex Domains, Trans. Amer. Math. Soc. vol. 348 (1996), 411-479.

[17] M. Engliš, A Forelli-Rudin contruction and asymptotics of weighted Bergman kernels, J. Func. Anal. 177 (2000), 257-281.

[18] M. Engliš, The asymptotics of a Laplace integral on a Kähler manifold, J. Reine Angew. Math. 528 (2000), 1-39. 
[19] S. Ji, Inequality for distortion function of invertible sheaves on Abelian varieties, Duke Math. J. 58 (1989), 657-667.

[20] G. R. Kempf, Metric on invertible sheaves on abelian varieties, Topics in algebraic geometry (Guanajuato) (1989).

[21] A. Loi, The Tian-Yau-Zelditch asymptotic expansion for real analytic Kähler metrics, Int. J. of Geom. Methods Mod. Phys. 1 (2004), 253-263.

[22] A. Loi, A Laplace integral, the T-Y-Z expansion and Berezin's transform on a Kaehler manifold, Int. J. of Geom. Methods Mod. Phys. 2 (2005), 359-371.

[23] A. Loi, Regular quantizations of Kähler manifolds and constant scalar curvature metrics, J. Geom. Phys. 53 (2005), 354-364.

[24] A. Loi, Bergman and balanced metrics on complex manifolds, Int. J. of Geom. Methods Mod. Phys. 2 (2005), 553-561.

[25] A. Loi, Calabi's diastasis function for Hermitian symmetric spaces, Differential Geom. Appl. 24 (2006), 311-319.

[26] A. Loi, Balanced metrics on $\mathbb{C}^{n}$, to appear in J. Geom. Phys.

[27] A. Loi, Regular quantizations and covering maps, to appear in Geom. Dedicata.

[28] Z. Lu, On the lower terms of the asymptotic expansion of Tian-Yau-Zelditch, Amer. J. Math. 122 (2000), 235-273.

[29] A. Odzijewicz, On reproducing kernels and quantization of states, Commun. Math. Phys. 114 (1988), 577597.

[30] A. Odzijewicz, Coherent states and geometric quantization, Commun. Math. Phys. 150 (1992) 385413.

[31] I. M. Mladenov and V. V. Tsanov, Reduction in stages and complete quantization of the MICKepler problem J. Phys. A 32 (1999), 3779-3791

[32] J. H. Rawnsley, A nonunitary pairing of polarizations for the Kepler problem, Trans. Amer. Math. Soc. 250 (1979), 167-180.

[33] J. H. Rawnsley, Coherent states and Kähler manifolds, The Quarterly Journal of Mathematics (1977), 403-415.

[34] W. D. Ruan, Canonical coordinates and Bergmann metrics, Comm. in Anal. and Geom. (1998), 589-631.

[35] M. Shubin, Pseudodifferential operators and spectral theory, Second edition, Springer-Verlag, Berlin.

[36] J. Sjöstrand, Singularités analytiques microlocales, Astérisque, 95 (1982) 1-166.

[37] J. M. Souriau, Sur la varie'te' de Kepler, Symposia Mathematica XIV, Academic Press (1974).

[38] G. Tian, On a set of polarized Kähler metrics on algebraic manifolds, J. Diff. Geometry 32 (1990), 99-130.

[39] S. Zelditch, Szegö Kernels and a Theorem of Tian, Internat. Math. Res. Notices 6 (1998), $317-331$.

[40] S. Zhang, Heights and reductions of semi-stable varieties, Comp. Math. 104 (1996), 77-105. 